\title{
EL RETO DE LA MOVILIDAD DOCENTE Y LOS ESTUDIOS GENERALES: UN ESTUDIO EN DOS ETAPAS
}

The challenge of teacher mobility and general studies: a study in two stages

\section{María Elena Córdoba Waldemiro Vélez}

Resumen: El punto de partida de este estudio es el programa de Movilidad Profesoral con docentes de Estudios Generales, que vincula tres Universidades de diversos países. Las tres ofrecen Estudios Generales en su propuesta educativa, dos desde hace varios años (Universidad de Puerto Rico y Universidad Pontificia Católica del Perú) y una mucho más reciente (Instituto Tecnológico de Santo Domingo). El estudio, del cual se presentan los resultados de la primera etapa, consiste en evaluar el impacto del programa de movilidad en la formación integral de estudiantes pertenecientes a las universidades participantes. Además, permitió tener una aproximación a las características de los programas de movilidad en las tres universidades, su filosofía y alcances. Por otra parte abordó la repercusión de los intercambios de docentes de Estudios Generales respecto a la interculturalidad y las estrategias docentes que utilizan en cada universidad para trabajar contenidos similares o problemáticas particulares. El estudio se subdivide en dos etapas, cada una de las cuales aportará sus propios resultados. Esta ponencia corresponde a los resultados de la primera etapa. Será en la segunda etapa en la que se hará un acercamiento más detallado a la evaluación del efecto que tienen estos programas de movilidad en la formación integral de los estudiantes.

Palabras clave: Movilidad docente, estudios generales, formación integral, integración de conocimientos, multiculturalidad. 
Abstract: The starting point of the study is the Faculty Mobility program with professors of General Studies, linking three universities of various countries. All three offer General Studies in their educational proposal, two of them have offered these for several years (Universidad de Puerto Rico and Universidad Pontificia Católica del Perú), and one more recently (Instituto Tecnológico de Santo Domingo). The study, which presents the outcomes of its first phase, consists of evaluating the impact of the mobility program in the integral formation of students from the participating universities. In addition, it allowed having an approach to the characteristics of the mobility programs in the three universities, their philosophy and scope. On the other hand, it addressed the impact of exchanges of General Studies professors regarding interculturality and teaching strategies used in each university to work with similar contents and particular problems. The study is divided in two phases; each one will render their own findings. This paper corresponds to the results of the first phase. A more detailed approach to the assessment of the effect that these mobility programs have in the integral formation of students will be presented in the second phase.

Keywords: Faculty mobility, General Studies, integral formation, knowledge integration, multiculturality.

\section{Introducción}

En esta primera etapa de la Investigación sobre los programas de Movilidad Docente en Estudios Generales que se está realizando en tres instituciones universitarias (Instituto Tecnológico de Santo Domingo, Pontificia Universidad Católica del Perú y Universidad de Puerto Rico, Recinto de Río Piedras), nos propusimos sentar las bases para hacer un primer acercamiento a la evaluación del impacto de los mencionados programas en la formación integral 
de los y las estudiantes. Partimos de la hipótesis de que al impactar la capacitación de los docentes, proveyendo experiencias y conocimientos sobre la diversidad de ofrecimientos y prácticas en los Estudios Generales, así como promoviendo una mayor sensibilidad intercultual, éstos harán una mejor aportación a la formación integral del estudiantado.

Debemos destacar que las diferencias idiosincráticas y culturales, tanto en teoría, filosofía educativa y prácticas concretas en las tres Instituciones incluidas en nuestra investigación ha representado tanto una riqueza extraordinaria para el enriquecimiento intelectual de los participantes, como un reto significativo para los investigadores. Al evaluar los programas de Movilidad Docente (características, filosofía y alcances) en dichas Instituciones, y sobre todo al intentar comprender cómo los ponen en práctica, nos topamos con diferencias que podrían resultar muy significativas. Esto podrá comenzar a apreciarse más adelante en este artículo, a partir de las reflexiones de los participantes que hemos venido recogiendo en esta investigación.

El hecho de que nuestro estudio se centra en un programa de Movilidad Docente en los Estudios Generales (MDEG) nos permite reflexionar sobre el currículo, la filosofía e implantación de este importante componente curricular. Con eso en mente hemos desarrollado diversos instrumentos que nos permitirán comparar la concepción que tienen los docentes de tres Instituciones Universitarias que participaron del proyecto de Movilidad, sobre las particularidades de los ofrecimientos de Estudios Generales en los que ellos y ellas ejercen la docencia. En el desarrollo y aplicación de estos instrumentos -como podrá constarse en la sección de este informe dedicada a la metodología de la investigación-hemos prestado particular atención a la identidad central de los Estudios Generales, la integración del conocimiento. Por esta razón, consideramos que podremos hacer una aportación teórico-práctica al fortalecimiento de los Estudios Generales. 


\section{Metodología}

La metodología del estudio consistió en establecer una triangulación entre las universidades participantes. El diseño se fundamentó en una metodología mixta en la cual se utilizaron estrategias cualitativas y cuantitativas para la recolección y análisis de datos propios de ambos enfoques.

Según explica Kelle (2006) el diseño mixto de investigación puede cumplir funciones similares a un diseño secuencial, en tanto la parte cualitativa del diseño puede producir información que ayuda a entender las asociaciones estadísticas y elaborar explicaciones que resultan de los análisis estadísticos de la investigación. (p. 308). Este tipo de diseño reconoce la triangulación de datos, fuentes de información, marcos teóricos y conceptuales, metodologías y perspectivas de los investigadores.

Para esta etapa de la investigación, se incluyó un sondeo con el objetivo de detectar y recopilar, en caso de que existieran, documentos sobre movilidad docente en general y de estudios generales en particular.

Con los datos recopilados se construyó un resumen de características generales que recoge los principales elementos de la movilidad docente en cada universidad participante. Por otra parte, con la información correspondiente al análisis y selección bibliográfica, se identificaron categorías y subcategorías que dieron lugar a la construcción de los instrumentos utilizados para la recolección de datos.

El trabajo de campo tuvo varias etapas, una inicial de visitas a las universidades participantes. En estas se establecieron los alcances del programa, compromisos de las partes y responsabilidades de los docentes que participarían, así como la modalidad de selección de los mismos. 
Con la información revisada se construyó el Programa de Movilidad Docente de Estudios Generales (MDEG), el cual sirve de marco para establecer los acuerdos con las instituciones participantes, a la vez que se definieron compromisos de los docentes y actividades propuestas durante las etapas de la estancia en la universidad visitada.

En estas visitas a las universidades, se realizaron entrevistas a Decanos, Directores de Estudios Generales y Encargados de Movilidad. Se efectuaron observaciones de actividades académicas, clases de Estudios Generales y colaboración en el proceso de selección de docentes que participarían en el proyecto.

El trabajo de campo incluía un pre-test, cuya población estuvo compuesta por seis docentes seleccionados, dos de cada país participante. Así también conforman la población los grupos de estudiantes de una sesión de cada docente. El pre-test se realizó mediante:

- Observación directa no participante en una de las clases de cada docente elegido para el programa de movilidad. Las clases observadas se seleccionaron aleatoriamente, y su finalidad consistía en consignar las estrategias utilizadas y características de la docencia en relación a la formación integral de sus estudiantes. Datos estos que se levantaron en una rúbrica construida para tal fin.

- Entrevista semiestructurada a cada docente participante a fin de obtener información respecto a su opinión de los Estudios Generales, la docencia en los mismos y las expectativas respecto a su participación en el proyecto de movilidad.

- Autoevaluación de cada docente participante, para rescatar su percepción en aspectos específicos de los Estudios Generales y la incidencia que según ellos tienen en la formación integral del estudiantado. 
Cuestionario aplicado a las y los estudiantes de un curso de cada docente seleccionado, con el objetivo principal de recoger su opinión en relación a las características del accionar pedagógico del docente.

Cabe señalar que esta etapa del estudio se realizó durante el primer año del programa de Movilidad Docente de Estudios Generales, por lo tanto se centró en las actividades del pre-test, impresiones de docentes y estudiantes participantes en el proyecto, además de recoger datos preliminares del impacto en la formación integral del estudiantado.

\section{Resultados obtenidos en las diferentes dimensiones de esta primera etapa}

En este artículo, correspondiente a la primera etapa del estudio, se presenta un resumen de los datos relacionados con los primeros tres objetivos propuestos, los tres restantes serán objeto de análisis en la segunda etapa de este estudio.

\subsection{Resultado del objetivo 1}

Describir las características de los programas de movilidad docente en cada universidad participante.

Respecto a las características de los programas de movilidad docente, hay que resaltar en primer lugar, que no existe en ninguna de las universidades participantes en este proyecto, ni en ninguna de las analizadas para el estudio, un programa de movilidad, que involucre puntualmente a docentes de Estudios Generales.

En las tres universidades participantes, existe acuerdos interinstitucionales que posibilitan la estancia de docentes en universidades en otro país, con el objetivo de llevar a cabo proyectos de cooperación, estancias profesionales, estudios doctorales o posdoctorales, docencia y/o investigación. Los períodos de duración varían de 
acuerdo a los intereses del proyecto y en algunas oportunidades se llevan a cabo mediante becas.

En el programa de Movilidad Docente de Estudios Generales de INTEC, los acuerdos implican, entre otras cosas, que el número de docentes visitantes de cada universidad será correspondido recibiendo igual número de docentes en la universidad de origen. Así también existen compromisos particulares que asume cada participante, con intenciones específicas de fortalecer sus competencias docentes e interculturales, así como dar vida a dos objetivos de la Red Internacional de Estudios Generales (RIDEG), en ellos dice:

1. Multiplicar otras instancias de diálogo y colaboración entre nuestras instituciones, tales como: talleres, encuentros, intercambio de profesores y estudiantes, conferencias y proyectos de investigación; para compartir experiencias y perspectivas, potenciando así nuestros recursos...

2. Desarrollar y apoyar propuestas conjuntas para enriquecer la experiencia estudiantil y colaborar en la capacitación continua del profesorado e impulsar iniciativas de investigación colaborativa e interdisciplinaria.

Un punto de coincidencia en las universidades, consiste en que la movilidad comenzó para el estudiantado, y la movilidad docente inicialmente ocurría promovida por los departamentos académicos. Posteriormente, para institucionalizar esta práctica, y en algunos casos impulsados por la internacionalización, se comienzan a asentar y centralizar estos programas explicitando sus alcances y modalidades. La Universidad de Puerto Rico, tiene diversos proyectos de movilidad docente, cada uno con intereses y propuestas diferentes. La Pontificia Universidad Católica del Perú, tiene un área de cooperación que atiende proyectos de movilidad docente en sus diversas modalidades. En INTEC el programa de movilidad docente de Estudios Generales, nació a la par de este estudio y es responsabilidad del área de Desarrollo Curricular. 
Los requisitos para participar de programas de movilidad docente también varían en cada institución, sin embargo existe un punto de coincidencia en que la mayoría de los fondos o programas para docentes se dirigen a profesores contratados de tiempo completo. Aunque ocasionalmente pueden asignarse fondos para docentes que no están nombrados, sino que se desempeñan por asignatura, esto varía en función de para qué son los fondos.

Otros requisitos están vinculados a contar con un grado académico específico para poder postular, y la aceptación de la institución en la que hará la estancia. En el caso de la PUCP para las estancias posdoctorales también solicitan para el postulante, que presente un proyecto de investigación, a fin de evaluarlo y determinar si es elegido. En cualquiera de las ofertas de las tres universidades, un requisito indispensable es tener altas calificaciones académicas. Otro elemento que se toma en consideración es el nivel académico de la institución de destino, considerando los ranquin, por ejemplo.

Entre los programas que tiene la PUCP existe uno que provee fondos para traer visitantes de otros países, en cuyo caso se analiza el currículo del visitante, la institución de dónde viene, si existen acuerdos previos, etc. Respecto a los compromisos de los participantes, varían según el proyecto en el que se inscribe su movilidad, si por ejemplo se le otorga una beca para doctorado, firman un compromiso de retorno. En caso de viajar para participar en un evento académico, al regreso presentan un informe sobre la actividad.

En ninguno de los programas de movilidad docente revisados, existe como propósito del mismo, la vinculación explícita con las expectativas de mejora en la formación de los estudiantes, aunque en algunos se menciona tangencialmente, sin embargo más bien hacen referencia a preferir proyectos específicos que se vinculan con aspiraciones estratégicas para la Universidad, o que sean de nueva creación y por ello resulten de interés. 
Respecto a la evaluación y seguimiento posterior de estos programas, no existen diseños de evaluación en los programas revisados, sólo en los casos en que se solicita un informe puede verse si se concretó el propósito de la movilidad. Otro indicador que puede considerarse son las estadísticas vinculadas a cantidad de postulantes, períodos en los que se realizó el programa, retorno a la universidad de origen, etc. Sin embargo, no hay un sistema de evaluación y seguimiento propiamente dicho.

Es importante resaltar que en las teorías y fundamentaciones de programas de movilidad docente revisados para este estudio, no se explicita puntualmente que tengan una intención de repercutir en la formación integral del estudiantado, sino que más bien apuntan a la formación docente, el intercambio y cooperación de proyectos colaborativos.

La movilidad de docentes y de investigadores permite, por una parte, establecer relaciones fluidas entre grupos de investigación y departamentos universitarios, lo que contribuye al refuerzo de las actividades conjuntas, al intercambio de información y a la optimización de los recursos disponibles; por otra parte, favorece el conocimiento mutuo, la experiencia de trabajo en contextos diferentes y el establecimiento de conexiones que suelen perdurar en el tiempo. Sus beneficios son múltiples y este tipo de intercambios pueden hacer más por la creación de un espacio común, que otros modos de relación más formalizados, pero también más distantes. (Tiana, 2012)

\subsection{Resultado del objetivo 2}

Analizar las características de la filosofía institucional, estrategias docentes y mecanismos de evaluación utilizados en cada institución involucrada. 
Con intensión de explicitar las características principales de cada una de las instituciones participantes, a continuación se revisaron la misión, visión y objetivos estratégicos de cada institución participante.

Los componentes esenciales expresados por cada Universidad evidencian la intención de la formación universitaria, adentrándonos en sus propósitos de acuerdo a los fundamentos que declaran, presentan algunos puntos de coincidencias.

Estos aspectos están relacionados a elementos fundamentales en el Proyecto de Movilidad docente de Estudios Generales, ellos son; la formación integral del estudiantado, la internacionalización y la excelencia académica. Aunque no siempre están denominados de igual forma, pero de sus declaraciones se deducen puntualmente los tres componentes. Otro elemento a considerar es que en las tres Universidades se cuenta con Estudios Generales como parte de su programa de estudios, cabe señalar que en INTEC no se llaman Estudios Generales en el pensum, sin embargo, es sólo una diferencia de nombre.

Es necesario tomar en cuenta que existen para cada uno de los elementos mencionados, diversas interpretaciones.

En este estudio, se partió de la concepción de formación Integral como el "proceso continuo, permanente y participativo que busca desarrollar armónica y coherentemente todas y cada una de las dimensiones del ser humano (ética, espiritual, cognitiva, afectiva, comunicativa, estética, corporal, y socio-política), a fin de lograr su realización plena en la sociedad. Es decir, vemos el ser humano como uno y a la vez pluridimensional, bien diverso como el cuerpo humano y a la vez plenamente integrado y articulado en una unidad". (Jornadas Docentes 2008).

La Internacionalización se ha convertido en un elemento que favorece el cambio a nivel universitario, independientemente de la interpretación particular que tenga. Originalmente implicaba proyectos de desarrollo internacionales, 
hoy las interpretaciones más comunes pueden agruparse en que para algunas universidades la internacionalización implica una serie de actividades como los traslados académicos de estudiantes y docentes, redes internacionales, proyectos conjuntos e investigaciones colaborativas. En algunos casos se refiere a la transmisión de educación a otros países, como por ejemplo la modalidad de franquicias, en modalidad presencial o a distancia. En otras instituciones se le concibe como la incorporación en el currículo y en el proceso enseñanza aprendizaje de la dimensión internacional, intercultural y global. (Knight, J. 2010)

En INTEC se declara en su estrategia institucional 2017, el redimensionamiento internacional de la gestión del currículo, indicando que hasta la fecha sólo corresponde a las alianzas internacionales circunscritas a actividades de cooperación que incluyen intercambios de estudiantes, programas de doble titulación y colaboración en proyectos de investigación conjunta.

Los propósitos indicados en la excelencia académica, hacen referencia a buscar siempre los más altos estándares de calidad en cada una de las actividades, y procesos que realiza. Es decir que la calidad de las ideas, los principios y actuaciones de los miembros de la comunidad académica, se sitúen en estándares que superan el funcionamiento regular, demostrando aspiraciones cada vez mejores en su desempeño.

\subsection{Resultado del objetivo 3}

Determinar los aspectos de la multiculturalidad que se ven involucrados en los participantes del programa.

Los programas de movilidad del profesorado, que posibilitan la estancia de docentes en universidades extranjeras, permiten la elaboración de trabajos y programas conjuntos de docencia e inves- 
tigación, lo que redunda en un mayor acercamiento de las comunidades académicas y en última instancia en un hermanamiento a nivel universitario que siempre implica mejores y más estrechas relaciones culturales.

A partir de la literatura relevante se puede comprobar que los Estudios Generales han sido y son los principales responsable de promover la conciencia ciudadana en los estudiantes universitarios. Pero no una ciudadanía excluyente y con pretensiones homogeneizadoras, sino a una ciudadanía intercultural y compleja. (Tubino, 2003)

Para dar respuesta a este objetivo, se tomaron en consideración las opiniones recogidos en tres de las preguntas hechas a los docentes antes de realizaran el viaje al país seleccionado.

Las preguntas son:

- ¿Cuán importante considera que es la comparación de diversas manifestaciones culturales?

- ¿Cuáles son las expectativas que tiene respecto a su participación en este programa de Movilidad docente?

- ¿Usted cree que esta experiencia podría impactar o cambiar en algo su docencia?

Probablemente por ser docentes de Estudios Generales, existe bastante coincidencia respecto a la importancia de la diversidad cultural. El grupo de docentes seleccionados, manifestó que la comprensión del entramado cultural es vital en la tarea docente. "Es la mejor forma de entender al otro y el respeto a la diversidad". La deconstrucción de verdades absolutas, atrapadas en modelos históricos o culturales, puede verse en los mismos docentes. En ocasiones les resulta difícil manejar situaciones cada vez más comunes, de tener estudiantes de diversas nacionalidades y culturas, en una misma clase. 
"Esto es algo que los docentes deberíamos saber manejar, pero es difícil aprenderlo en un libro. Compartir con colegas de culturas diferentes, nos puede ayudar a entender la diversidad desde dentro".

En todas las entrevistas manifestaron la gran importancia de la cultura para entender la historia, la antropología, la política, el pensamiento contemporáneo, etc. Así como también hicieron hincapié en que ya no es posible tal comprensión si sólo nos limitamos a profundizar una cultura y de las demás, sólo tenemos aproximaciones. Es necesario un abordaje que enriquezca el enfoque multicultural. En este caso, el programa de movilidad que estamos analizando, parece ser una forma de nutrir ese enfoque.

"Nosotras tenemos que enseñar que no somos todos iguales... Así que este intercambio de culturas entre nosotros realmente nos permitirá comprender la multiculturalidad que enseñamos".

Consultados sobre sus expectativas al participar en este programa de movilidad, las respuestas también coincidentes en lo esencial, hicieron referencia a que sólo el acercarse a las expectativas del programa y las lecturas proporcionadas, ya les habían brindado una oportunidad de aprendizaje de algunos elementos no considerados anteriormente de la misma forma, como por ejemplo el enfoque transdisciplinario en los Estudios Generales.

“...me he dado cuenta que realmente en este mundo globalizado, de tanta complejidad, tanto para el conocimiento, como para la conducta del ser humano se necesita esa formación integral, esa formación que nos lleve a que seamos mejores seres humanos, y es parte de mis expectativas con este programa, hacer cada vez mejor mi trabajo".

“...Lo cultural está por todas partes, porque la universidad está viva. Esto también aplica para nosotros, este programa de movilidad, nos brindará esa oportunidad". 
Otro punto de coincidencia sobre este aspecto, fue el comprobar que la multiculturalidad es vista por las y los docentes seleccionados, como una necesidad.

También expresaron lo interesante que les resultará ver el "caso peruano" o el "fenómeno dominico-haitiano", o el "caso portorriqueño" directamente, con la posibilidad de observar cómo se enseña en esos lugares así como de qué manera abordan la diversidad y la formación integral en otro lugar.

$\mathrm{Al}$ consultarles si consideraban que esta experiencia podría cambiar o impactar en algo su docencia, en forma unánime dijeron que sí. "Mi docencia va a cambiar, de acuerdo a las herramientas que estoy aprendiendo y las que aprenderé cuando viaje, seguro que vamos a formar mejores profesionales, porque para que sean mejores profesionales, las y los docentes tenemos que cambiar".

“...mi idea básica es conocer la experiencia que los docentes de otro país están teniendo, ver en que puedo mejorar metodológicamente, en que puedo cambiar".

"Esta es la forma en que podemos aprender docencia, quienes no estudiamos pedagogía. ... de esta manera, sí podemos aprender a ser mejores docentes, esto debe ser presentado como una modalidad pedagógica de enseñar docencia".

"puedo haber leído sobre un lugar, pero otra cosa es recorrer su terreno, escuchar con ellos su música, comer con ellos sus comidas, sentarnos con estudiantes y observar cómo se dirigen a ellos, cómo enfrentan la enseñanza y la formación integral".

Las expectativas puestas en el programa son muchas ya que resulta muy interesante comprobar que un gran número de docentes, no sólo los que participaron en este programa, entienden que su formación, dada la naturaleza de los cambios en el mundo, en la educación y en la juventud, es precisamente compartiendo con pares, y si tienen la oportunidad de hacerlo en otros países, tanto mejor. 


\section{Conclusiones}

En este artículo presentamos los logros alcanzados a partir de tres objetivos que nos propusimos en nuestra investigación. En primer lugar, describimos las principales características de los programas de movilidad docente en cada una de las tres universidades que participaron en el proyecto inicial de movilidad docente en Estudios Generales; la Pontificia Universidad Católica del Perú (PUCP), la Universidad de Puerto Rico (UPR) y el Instituto Tecnológico de Santo Domingo (INTEC). En el proceso pudimos registrar las diferencias y similitudes entre éstas, así como el lugar e interés que le otorgan a los procesos de intercambio de docentes y estudiantes. Aquí los diversos énfasis en la investigación y los post-grados han resultado ser muy importantes. Es significativo, además, que aun cuando dos de las tres tienen larga experiencia con los Estudios Generales (una con más de 71 años y la otra con más de 40 años), ninguna había elaborado un proyecto de movilidad docente particularmente para este importante componente curricular.

En segundo lugar, analizamos las características de la filosofía institucional, las estrategias docentes en los Estudios Generales y los mecanismos de evaluación utilizados en este componente académico, en cada institución involucrada. Para ello consideramos sus documentos de misión, visión y objetivos estratégicos. En todos los casos, se establecía que las experiencias internacionales resultan sumamente enriquecedoras y que debían ser parte importante de sus ofrecimientos. Sin embargo, no se le otorgaba el énfasis que esperábamos al papel de éstas en la capacitación docente y su efecto en la formación integral del estudiante. De ahí que no se le diera prioridad a evaluar continuamente este aspecto de los resultados de la Movilidad. Por ello, la investigación que estamos desarrollando es de extraordinaria importancia, por la incidencia que podría tener en la generación de nuevas políticas institucionales que atiendan expresamente la mencionada prioridad, así como la construcción de instrumentos y mecanismos para llevarla a la práctica. 
En tercer lugar, consideramos el impacto que tendría la Movilidad en el desarrollo de una mayor sensibilidad multicultural en los participantes del programa. Las diversidades culturales de los países visitados, así como las particularidades de cada una de las Instituciones participantes, les permitieron a los docentes enriquecer su acervo de conocimientos, así como exponerse a patrones de conducta cotidiana diferentes a los de sus países de procedencia. Como era de esperar, los caribeños nos sentimos inicialmente desubicados en un país andino como el Perú, particularmente en Lima, una ciudad que no deja de sorprendernos, cuando por ejemplo nos dicen: "aquí nunca llueve". Así también en el Caribe, la lluvia, y los efectos que provoca en nuestra cotidianidad, no dejaba de sorprender a los peruanos. Por otro lado, aún con las similitudes y fraternidad histórica que podemos tener los puertorriqueños y dominicanos, las diferencias culturales, políticas y económicas que se han producido por la diferente relación de ambas con los Estados Unidos, no deben menospreciarse.

Esa exposición tanto a regímenes académicos diversos como a cotidianidades culturales sumamente ricas y desafiantes, provocó una mayor sensibilidad multicultural en los docentes que participaron del Proyecto de Movilidad, como pudo apreciarse claramente en algunos de sus testimonios que fueron recogidos en la sección anterior.

Una labor que a nuestro juicio representa una extraordinaria aportación de este artículo ha sido la elaboración de Instrumentos para evaluar todo el proceso de movilidad docente de Estudios Generales. Dichos instrumentos, y esto merece especial reconocimiento, se adentran en las características más definitorias de los ofrecimientos de este importante componente curricular: la amplitud de miras y la integración del conocimiento. Entendemos a que a partir de las preguntas presentadas, así como del orden y la integralidad que representan, los lectores podrán acercarse a un mejor entendimiento de lo que entendemos por Estudios Generales. 
La movilización de conocimientos en la sociedad: Un reto para los programas doctorales en la universidad pública latinoamericana

\section{Bibliografía}

Dewey, J. (2004). Democracia y educación. (6ta. Ed.). Buenos Aires: Losada.

Freedman, R. (2009). Noise Wars. Compulsory Media and Our Loss of Autonomy. New York: Algora Publishing.

Instituto Tecnológico de Santo Domingo. (2013). Estrategia institucional 2017. Santo Domingo: El autor.

Kelle, U. (2006) Combining qualitative and quantitative methods in research practice: purposes and advantages. Qualitative Research in Psychology, 3(4), 293-311.

Knight, J. (2010). Intenacionalización de la educación superior. Instituto de Ontario de Estudios en Educación: Universidad de Toronto.

Madarro, A. (2011). Redes de movilidad académica para la cooperación e integración regional en Iberoamérica. Revista Iberoamericana de Educación, 57, 71-107.

Marsh, C. \& G. Willis (2007). Curriculum: Alternative Approaches, Ongoing Issues (4ta. Ed.). Upper Saddle River. New Jersey: Pearson y Merrill Prentice Hall.

Martínez, M. Ríos E. \& Vélez W. (2012). La investigación institucional en las instituciones de Educación Superior en Puerto Rico: Una tipología de la Oficina de Investigación Institucional. San Juan, PR: CEDESP.

Mercado Común del Sur. (2011). Plan Estratégico del Sector Educativo. 2011-2015. Recuperado de http://edu.mercosur.int/es-ES/ plano-2011-2015.html

Ministerio de Educación de Argentina. (s. f.). Programa de Internacionalización de la Educación Superior y Cooperación Internacional. Buenos Aires: El autor. 
Morin, E. (1993). El método I: La naturaleza de la naturaleza. Madrid: Ediciones Cátedra.

Morin, E. (1999). El método III: El conocimiento del conocimiento. Madrid: Ediciones Cátedra.

Morin, E. (2001). El método IV: Las ideas. Madrid: Ediciones Cátedra.

Morin, E. (2003). Introducción al pensamiento complejo. Barcelona: Gedisa Editorial.

Nicolescu, B. (2002). Manifesto of Transdisciplinarity. Albany, New York: State University of New York Press.

Organización de Estados Iberoamericanos para la Educación, la Ciencia y la Cultura. (2012). Informe Anual del Programa Pablo Neruda. Madrid: Organización de Estados Iberoamericanos para la Educación, la Ciencia y la Cultura.

Pontificia Universidad Católica del Perú. (2013). Estrategia institucional 2017. Perú: Pontificia Universidad Católica del Perú.

Sebastián, J. (2002). Oportunidades e iniciativas para la cooperación iberoamericana en educación superior. Revista Iberoamericana de educación, N. 28, 197-229.

Sebastián, J. (2004). Cooperación e internacionalización de las universidades. Buenos Aires: Biblos.

Siufi, G. (2009, enero). Cooperación internacional e internacionalización de la educación superior. Nueva Época, 123-145.

Subirats, P. \& Vélez, W. (2010). La educación general en el Siglo XXI. (Ponencia presentada en el Primer Simposio Internacional de Estudios Generales). San Juan, Puerto Rico: Recinto de Río Piedras de la Universidad de Puerto Rico. 
Tiana, A. (2012). La contribución de la movilidad académica a la construcción de un espacio iberoamericano de educación superior. Revista Lusófona de Educaşão, (21), 53-68.

Tubino, F. (2003). Ciudadanías complejas y diversidad cultural. En N. Vigil y R. Zariquiey (Eds.). Ciudadanias inconclusas. El ejercicio de los derechos en sociedades asimétricas (Pp.167-191) Lima: Cooperación Alemana al Desarrollo y PUCP.

Universidad de Puerto Rico. (2016). Estrategia institucional 2016. San Juan, PR: el autor.

Volkwein, J. F. (2008). The foundations and evolution of Institutional Research. New York: Directions for Higher Education.

\section{María Elena Córdoba}

Es educadora investigadora. Actualmente se desempeña como directora de Desarrollo Curricular en el Instituto Tecnológico de Santo Domingo. Estudió Psicología en la Universidad Nacional de Córdoba, Argentina; cursó una maestría en Educación Social y Animación Sociocultural por la Universidad de Sevilla-INTEC; posee un doctorado en Educación por la Atlantic International University.

Ha sido asesora y consultora en Desarrollo Curricular y Formación Docente de varias universidades de México. Se desempeñó como docente de Metodología de la investigación en diversos programas de maestrías en el Instituto de Estudios 
Universitarios de México. Ha impartido numerosos cursos y talleres sobre elaboración de tesis y Metodología de la Investigación; también ha dirigido investigaciones sociales, educativas y psicológicas en México, Argentina y República Dominicana. En el campo de la Investigación tiene trabajos sobre el rol y la función docente, evaluación, currículo, formación y actualización del profesorado.

Email: maria.cordoba@intec.edu.do

\section{Waldemiro Vélez Cardona, Ph. D.}

Catedrático en el Departamento de Ciencias Sociales de la Facultad de Estudios Generales, Universidad de Puerto Rico y coordinador de la Red Internacional de Estudios Generales (RIDEG).

Email: waldemirov@hotmail.com

Recibido: 09-04-2015

Aprobado: 13-09-2015 